\title{
Acute Lower Gastrointestinal Bleeding
}

\author{
Rakesh Navuluri, M.D. ${ }^{1}$ Lisa Kang, M.D. ${ }^{1}$ Jay Patel, M.D. ${ }^{1}$ Thuong Van Ha, M.D. ${ }^{1}$ \\ ${ }^{1}$ Department of Radiology, the University of Chicago Medical Center, \\ Chicago, Illinois \\ Address for correspondence and reprint requests Rakesh Navuluri, M.D., \\ Department of Radiology, 5841 S. Maryland Ave., MC2026, Chicago, IL \\ 60637 (e-mail: rnavuluri@radiology.bsd.uchicago.edu).
}

Semin Intervent Radiol 2012;29:178-186

\begin{abstract}
Keywords

- embolization

- Gl bleeding

- emergent

The annual incidence of lower gastrointestinal bleeding (LGIB) is $\sim 20.5$ in 100,000 in the general Western population and results in 1 to $2 \%$ of hospital emergencies. When medical management and endoscopic therapy are inadequate in cases of acute LGIB, endovascular intervention can be lifesaving. In these emergent situations it is important for the interventional radiologist to be well versed in the multidisciplinary preangiographic work-up, the angiographic presentations of LGIB, and the endovascular therapeutic options. We describe a case of LGIB managed with endovascular embolization and detail the angiographic techniques used, followed by a detailed discussion of the various treatment approaches to LGIB.
\end{abstract}

Objectives: Upon completion of this article, the reader will be able to review the current diagnostic and treatment algorithms for acute lower gastrointestinal hemorrhage.

Accreditation: Tufts University School of Medicine is accredited by the Accreditation Council for Continuing Medical Education to provide continuing medical education for physicians.

Credit: Tufts University School of Medicine designates this journal-based CME activity for a maximum of $\mathbf{1}$ AMA PRA Category 1 Credit $^{\mathrm{TM}}$. Physicians should claim only the credit commensurate with the extent of their participation in the activity.

\section{Case Presentation}

A 63-year-old woman with multiple medical diagnoses including prior stroke and hypertension presented to an outside institution with bright red blood per rectum. A total of 15 units of packed red blood cells ( $\mathrm{pRBCs}$ ) were given for massive gastrointestinal (GI) bleeding before the patient presented to our institution. She was found to be anemic with hemoglobin of $9.3 \mathrm{~g} / \mathrm{dL}$. Coagulation parameters were normal. Flexible sigmoidoscopy performed by the gastroenterology service demonstrated a Dieulafoy lesion in the distal rectum, $\sim 5 \mathrm{~cm}$ from the anal verge. Active hemorrhage from a protruding arteriole was noted within this lesion (-Fig. 1). To achieve hemostasis, $5 \mathrm{~mL}$ of 1:10,000 epinephrine was initially injected endoscopically into the lesion. A bipolar probe was then used for thermal coagulation and tissue destruction. Additionally, two hemostatic clips were placed across the lesion, but the bleeding persisted. Following endoscopic therapy and initial partial hemostasis, the patient's lower gastrointestinal bleeding (LGIB) recurred, requiring transfusion of 15 additional units of pRBCs.

\section{Diagnosis}

Rectal Dieulafoy lesion refractory to medical management and endoscopic intervention.

\section{Treatment/Equipment}

The right common femoral artery was accessed using a micropuncture system (Cook, Bloomington, IN), and a 5F vascular sheath (Terumo, Elkton, MD) was placed. A multi-sidehole infusion catheter (Cook) was advanced into the abdominal aorta over a guidewire. An aortogram, performed at an injection rate of $20 \mathrm{~mL} /$ second for $30 \mathrm{~mL}$, demonstrated a normalappearing aorta and mesenteric arterial anatomy. Using a 15$\mathrm{mm} \mathrm{J}$ wire (Cook) and a 5F RIM catheter (Cook), the inferior mesenteric artery (IMA) was selected under fluoroscopic guidance. An IMA angiogram, performed at an injection rate of $3 \mathrm{~mL} /$ second for $12 \mathrm{~mL}$, revealed active extravasation from an enlarged branch of the superior rectal artery (-Fig. 2). Using a coaxial system, the superior rectal artery was selected with a 3F Renegade STC microcatheter (Boston Scientific, Natick, MA) and Transend EX Floppy 0.014-inch microwire
Issue Theme Emergency IR; Guest Editor, Thuong G. Van Ha, M.D.
Copyright @ 2012 by Thieme Medical Publishers, Inc., 333 Seventh Avenue, New York, NY 10001, USA. Tel: +1(212) 584-4662.
DOI http://dx.doi.org/ $10.1055 / \mathrm{s}-0032-1326926$. ISSN 0739-9529. 


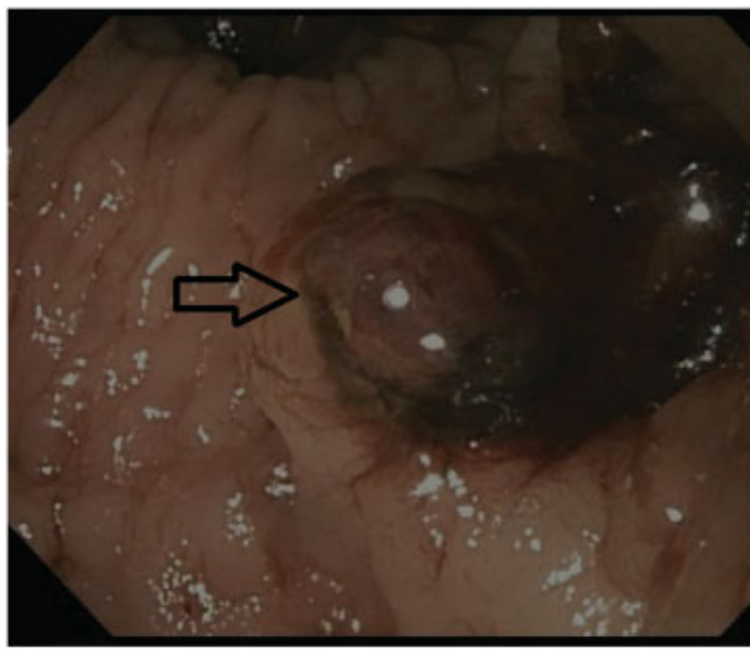

Figure 1 Colonoscopy image of a Dieulafoy lesion within the distal rectum $\sim 5 \mathrm{~cm}$ from the anal verge (arrow).

(Boston Scientific). After confirming catheter positioning with superselective angiography (-Fig. 3), embolization was performed using $6 \mathrm{~mm} \times 20 \mathrm{~cm}$ and $6 \mathrm{~mm} \times 10 \mathrm{~cm}$ Interlock microcoils (Boston Scientific), followed by gelatin foam pledgets (Gelfoam, Pfizer, New York, NY). Postembolization angiogram of the IMA confirmed resolution of contrast extravasation (-Fig. 4). A follow-up pelvic arteriogram was performed using a multiple side hole infusion catheter, and no additional sources of rectal bleeding were identified.

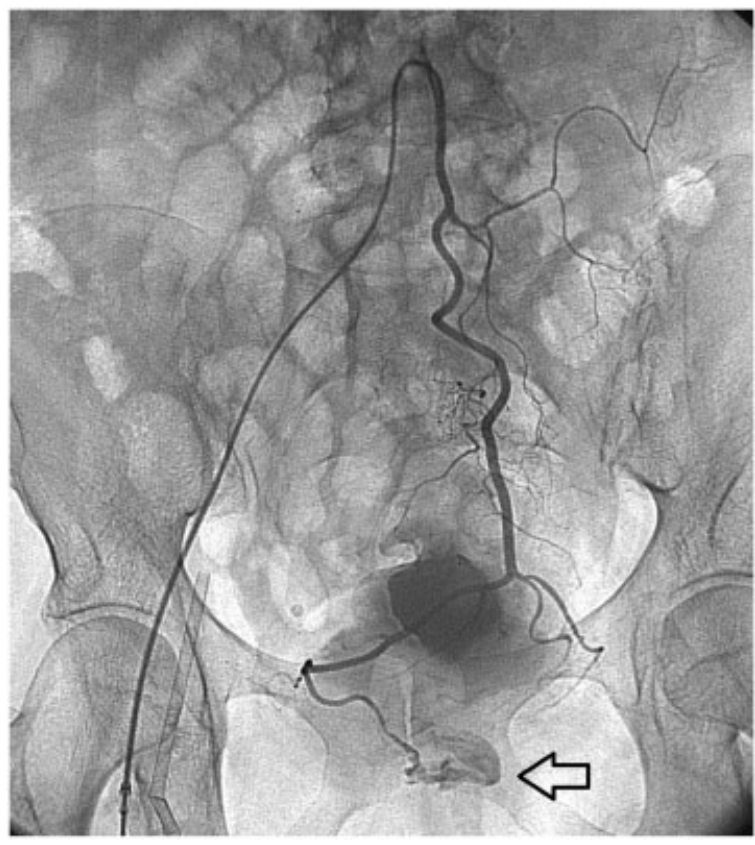

Figure 2 Inferior mesenteric artery arteriogram posteroanterior projection, midarterial phase, demonstrating active extravasation with pooling of contrast (arrow) arising from a prominent right-sided branch of the superior rectal artery.

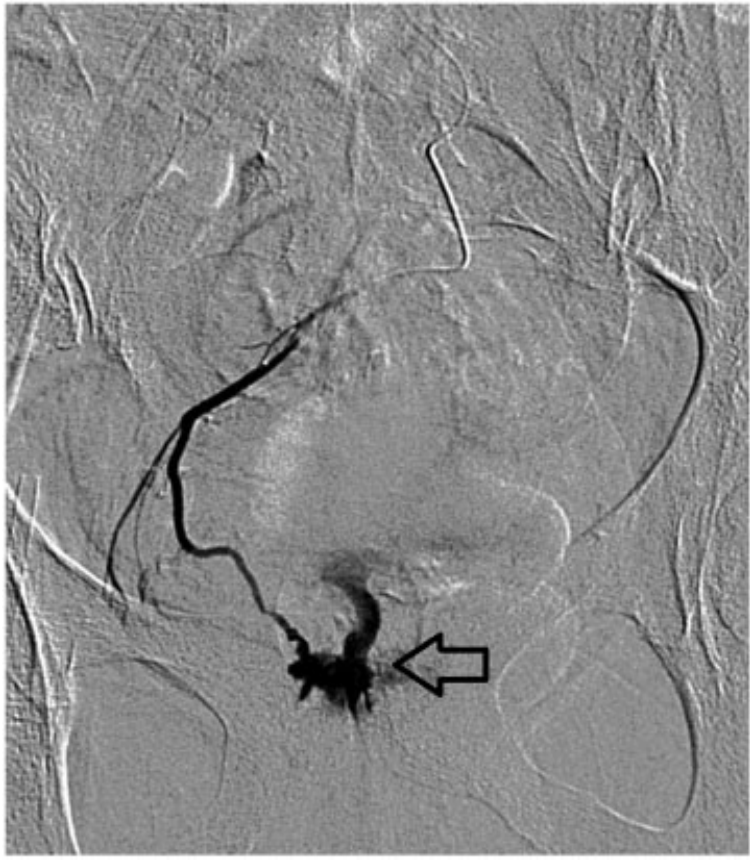

Figure 3 Superior rectal artery angiogram, posteroanterior projection, early arterial phase, with superselection of the prominent rightsided branch using a microcatheter. Gross contrast extravasation from this vessel is confirmed (arrow).

After endovascular therapy, the patient's hematochezia resolved and her hemoglobin level stabilized. No further intervention was necessary prior to discharge.

\section{Discussion}

\section{Background}

LGIB is defined as any bleeding source distal to the ligament of Treitz. ${ }^{1,2}$ Approximately $21 \%$ of patients with GI bleeding have a lower GI source, ${ }^{2}$ with an incidence in the U.S. population between 20.5 to 27 per 100,000 adults. ${ }^{1,3,4}$ This incidence increases $>200$-fold from the third to the ninth decade of life, ${ }^{1,3,5}$ paralleling the increase in prevalence of colonic diverticulosis and angiodysplasia. Men are afflicted more frequently than women. ${ }^{1}$ Acute LGIB is responsible for 1 to $2 \%$ of hospital emergencies, with $15 \%$ presenting as massive life-threatening hemorrhage ${ }^{5}$ and 5\% requiring emergent surgery. ${ }^{6}$ The overall mortality rate is $\sim 2$ to $4 \%{ }^{1}$

With rare exception, LGIB is arterial in origin. Causes of LGIB can be categorized based on their location in the small or large intestine. Acute colonic hemorrhage is most commonly secondary to diverticulosis or angiodysplasia. ${ }^{3}$ Other etiologies include colitis (inflammatory, infectious, ischemic, radiation), neoplasm, varices, and trauma. The most common causes of small bowel hemorrhage include enteritis, neoplasm, arteriovenous malformation, ulcer, and trauma. ${ }^{1}$

Dieulafoy lesions cause $\sim 5 \%$ of all GI hemorrhages, ${ }^{7}$ but they are a rare cause of acute LGIB. ${ }^{1}$ They are characterized by a mucosal defect through which a submucosal arteriole is exposed. Although endoscopic treatment is successful in achieving hemostasis in $90 \%$ of cases, ${ }^{8}$ endovascular 


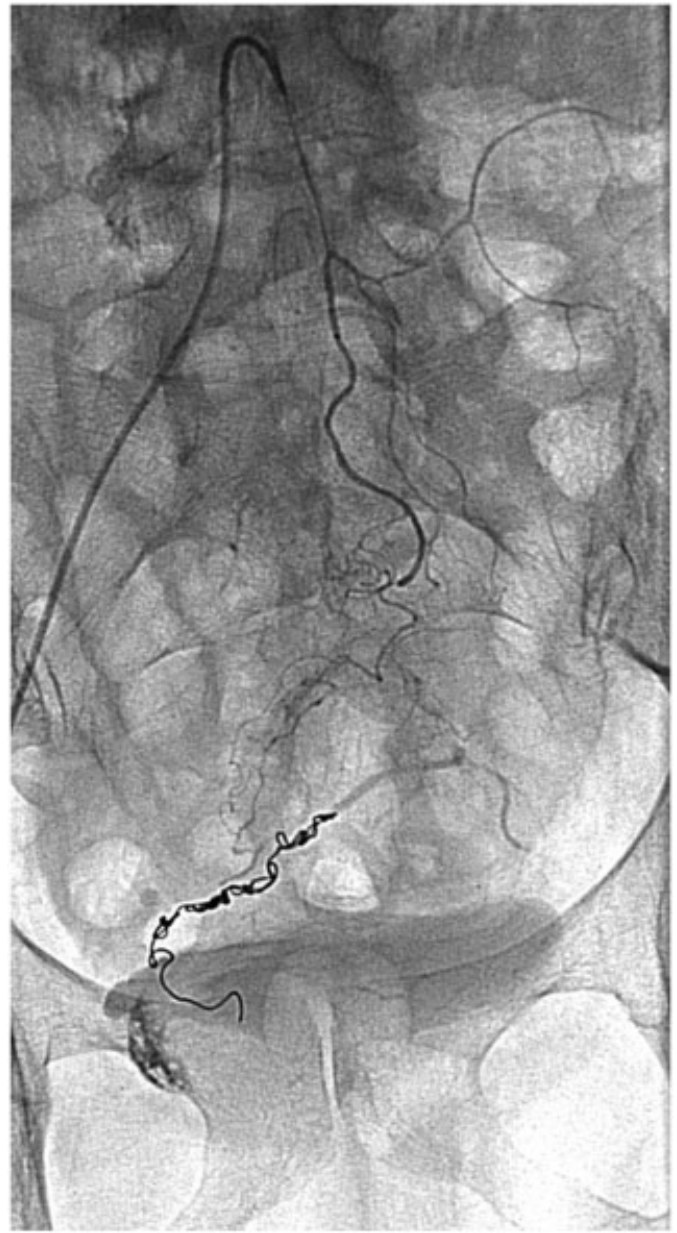

Figure 4 Inferior mesenteric artery arteriogram, posteroanterior projection, midarterial phase, with resolution of active extravasation after coil embolization of a right-sided branch of the superior rectal artery.

treatment can be used to control refractory bleeding, as demonstrated in the previous case.

\section{Symptoms}

Hematochezia is the typical presenting sign of LGIB. Rarely, it can be secondary to upper GI hemorrhage if there is brisk bleeding or quick bowel transit time of the intraluminal blood. Conversely, melena may present in patients with LGIB when there is slow bowel transit time. In a study of LGIB, Laine and Shah found that $15 \%$ of patients with clinically significant hematochezia, no symptoms of upper GI bleeding, and a negative nasogastric aspirate were found to in fact have an upper GI source of bleeding. ${ }^{9}$

Anemia and hemodynamic instability are clinical findings that can also be seen with LGIB. A loss of $<200 \mathrm{~mL}$ of blood usually has no effect on vital signs, whereas losses $>800 \mathrm{~mL}$ classically result in a decrease in blood pressure by $10 \mathrm{~mm} \mathrm{Hg}$ and an increase in heart rate of 10 beats per minute. Blood loss $>1500 \mathrm{~mL}$ may result in hemodynamic shock. ${ }^{1}$

\section{Algorithm}

Current therapeutic algorithms (-Fig. 5) for acute LGIB call for immediate medical stabilization followed by endoscopic

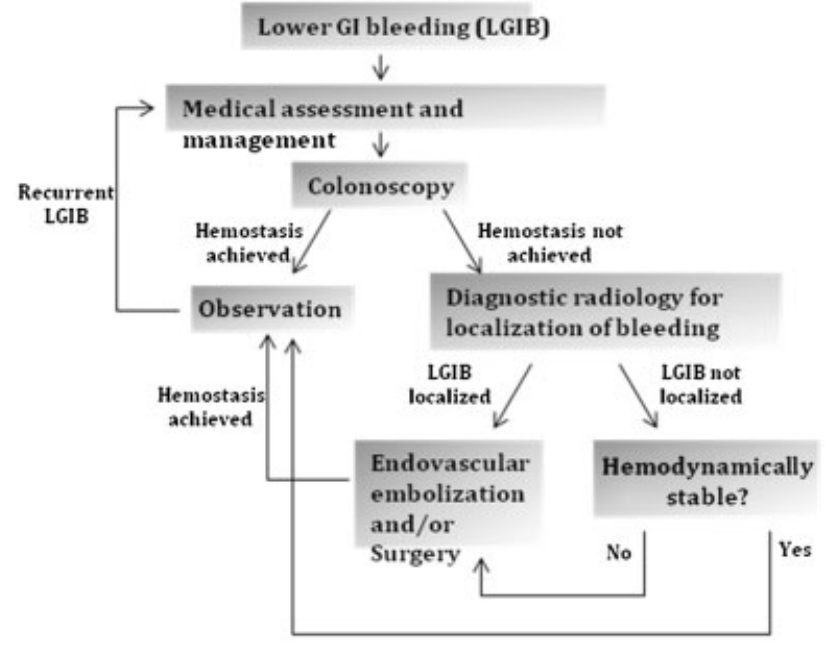

Figure 5 Algorithm for lower gastrointestinal bleeding management and treatment.

diagnosis and intervention. Patients who cannot undergo colonoscopy or those in whom the GI bleeding source remains unclear after endoscopy should be referred to diagnostic radiology for further evaluation by nuclear medicine or contrast-enhanced computed tomography (CT). Cases refractory to endoscopic intervention and those cases with positive diagnostic imaging results should be referred for endovascular intervention. Cases with negative diagnostic imaging results may be referred for conventional angiography with possible intervention depending on the clinical scenario. Surgery is the final therapeutic option.

\section{Medical Management}

Medical management is the first line treatment for LGIB. Stabilization of blood pressure with fluid resuscitation is the immediate goal. Patients with hemodynamically significant bleeding are immediately typed and crossed to facilitate fast and safe transfusion of blood products when needed. Any existing coagulopathy (international normalized ratio [INR] $>1.5$ or $<50,000$ platelets $/ \mu \mathrm{L}$ ) should be corrected. ${ }^{1}$ It is important to note that units of PRBC do not contain the factors and platelets needed for thrombogenesis, and if these are not also replaced by specifically giving platelets or freshfrozen plasma, a dilutional coagulopathy will result. An estimated 10 to $15 \%$ of cases require intervention beyond medical management to control bleeding. ${ }^{10}$

\section{Endoscopy}

After failed medical management, endoscopy is the diagnostic and therapeutic procedure of choice. Compared with upper endoscopy, colonoscopy suffers from low detection rates of bleeding lesions. Inadequate bowel prep in the emergent setting and presence of blood clots hinders the ability to visualize sources of bleeding. Additionally, profuse bleeding also obscures visualization of the offending lesion. A definitive source of bleeding is found in only $42 \%$ of urgent colonoscopies in patients with ongoing bleeding, despite purge colon preparation. ${ }^{11}$ 
No single lower GI endoscopic technique can both image the entire length of the lower GI tract and allow for intervention. Colonoscopy is limited to the colon and terminal ileum, and double-balloon enteroscopy allows for examination of only the proximal 50 to $120 \mathrm{~cm}$ of the jejunum. Retrograde double-balloon enteroscopy via a rectal approach is similarly limited in its reach. ${ }^{1}$ Capsule endoscopy can visualize the entirety of the small intestines, providing greater sensitivity and specificity for a LGIB source than enteroscopy, but it does not offer the option of intervention.

Moreover, these endoscopic techniques are not universally available, require experienced operators, and can be prohibitively time consuming. Practically speaking, capsule endoscopy and double-balloon enteroscopy are not feasible in the setting of emergent LGIB. Even urgent purge preparation for colonoscopy requires 3 to 4 hours to achieve adequate colon evacuation. 4,11

Endoscopic hemostasis can be achieved via epinephrine injection, thermocoagulation, and/or clipping/banding. Hemostatic clip (Hemoclip) placement or band ligation is considered the first option in the management of a Dieulafoy lesion. ${ }^{12}$ In Dieulafoy lesions, rebleeding after mechanical hemostasis occurs in $14.7 \%$ and $3.1 \%$ of cases treated with Hemoclips and banding, respectively. ${ }^{13}$ Rebleeding after endoscopically achieved hemostasis in general is reported to occur in 12 to $14 \%$ of patients. ${ }^{11}$ One ancillary benefit of Hemoclip placement is that it can direct subsequent endovascular intervention to the area of concern because of its radio-opacity.

\section{Localization}

Localization of bleeding prior to angiography is important for several reasons. First, it decreases procedure time by allowing for a more targeted approach. This consequently reduces radiation exposure as well as contrast load used during angiography. Minimizing contrast dose is critical in patients with borderline azotemia. Localization has also been shown to directly impact the success rate of endovascular therapy. ${ }^{14}$

If the source of bleeding cannot be localized, angiography may be deferred. However, in the setting of hemodynamic instability or in patients requiring $>5$ units of pRBCs, angiography should be performed immediately. In these circumstances, angiography is the most practical diagnostic tool because it offers the option of concomitant therapy. ${ }^{15}$ It is worth noting, however, that angiography is not very sensitive in detecting LGIB and has been reported to be as low as 35\% of cases. $^{16}$

\section{Nuclear Medicine}

If the source of bleeding is not identified endoscopically, radionuclide scintigraphy should be performed to localize the bleeding site. Gunderman et al found that a positive bleeding scan increased the likelihood of a positive angiogram from $22 \%$ to $53 \% .{ }^{14}$ A technetium 99 m-labeled red blood cell scan can detect bleeding rates as low as $0.2 \mathrm{~mL} /$ minute, compared with $0.5 \mathrm{~mL} /$ minute for angiography, and can be particularly useful in the setting of intermittent GI bleeding. Whereas angiography provides a 10 -second glimpse of the mesenteric circulation, a bleeding scan allows for interrogation over a multiple-hour window, thereby increasing sensitivity for LGIB detection. Although precise localization is not always possible due to the low resolution of planar imaging, bleeding scans are generally sufficient enough to aid in subsequent endovascular intervention. In patients with hemodynamic instability, studies with an immediate tracer blush are more likely to have a positive angiogram than studies with a delayed blush. ${ }^{16}$ Of note, positive scintigrams can occur in rare cases of variceal LGIB. We have encountered one case of massive small bowel variceal bleeding initially diagnosed as a lower GI arterial bleed in which the nuclear medicine bleeding scan was floridly positive.

\section{Computed Tomography Angiography}

Preparation time for radionuclide scintigraphy can make it impractical in the setting of emergent LGIB. More recently CT angiography (CTA) has found a role in the diagnosis and localization of GI hemorrhage. The examination can usually be performed more expeditiously than radionuclide scintigraphy, and modern multidetector scanners allow for the rapid acquisition of images. Marti et al found CTA to have a sensitivity of $100 \%$ and a specificity of $96 \%$ in localizing LGIB. ${ }^{17}$ CTA may even have a higher sensitivity than conventional angiography because it can detect lesions that are not bleeding at the time of the study. CTA protocols typically call for a noncontrast series followed by intravenous contrastenhanced series in arterial and delayed phases. This provides up to a 90-second window of time to evaluate for contrast extravasation, depending on the timing of the delayed series. Enteric contrast is not given secondary to its potential to obscure GI intraluminal bleeding.

An additional advantage of CTA is that the cross-sectional data provided gives insight into variant vascular anatomy prior to catheterization. Similarly, it can better characterize the inciting lesion in planning for treatment. If, for example, a mass lesion is seen, particle embolization may be performed where it might otherwise be avoided in the treatment of GI hemorrhage to avoid bowel necrosis. It has been suggested by some authors that CTA be used as the first step in the evaluation of patients with hemodynamically significant acute LGIB. ${ }^{17}$

The primary drawback of CTA is the necessity of intravenous iodinated contrast, which may elicit a hypersensitivity reaction or cause renal injury. ${ }^{18}$ This can be particularly concerning when considering the additional contrast required in a subsequent endovascular procedure.

Once active hemorrhage is documented and localized with a diagnostic radiology study, the patient should be transferred immediately to the angiography suite. At our institution, we strive to perform angiography within 1 hour of radiological diagnosis of LGIB.

\section{Preangiography Laboratory Work-Up}

Before angiography, the patient's renal and coagulation statuses should be assessed. Elevated prothrombin time/INR and partial thromboplastin time, as well as thrombocytopenia, should be corrected. Although there is no evidence to support 
a minimal threshold, several studies have demonstrated preinterventional coagulopathy to have a negative effect on clinical success. ${ }^{19,20}$ This makes sense when considering that most embolic agents cause vessel occlusion by providing a scaffold for thrombus formation rather than by pure mechanical obstruction. Exceptions to this are glue and Onyx (Micro Therapeutics, Irvine, CA) as discussed later.

\section{Angiography Technique}

The right common femoral artery is the default access site for mesenteric angiography. If marked angulation of the mesenteric vessels is documented prior to the procedure, one may consider a brachial artery access for improved mechanical advantage in manipulating catheters. A guiding catheter can also help in accessing tortuous or stenotic mesenteric arteries. If no prior imaging is available, an aortogram, in anteroposterior and/or lateral views, can be performed to map the origins of the mesenteric vessels. At our institution, a Rosch celiac 1 (RC-1, Cook) or visceral selective 1 (VS1, Cook) catheter is preferred for selecting the superior mesenteric artery (SMA). The injection rate for a superior mesenteric angiogram is typically $5 \mathrm{~mL} /$ second for a total volume of 20 to $25 \mathrm{~mL}$. We use a Rosch inferior mesenteric (RIM) catheter (Cook) to select the IMA. The injection rate for an inferior mesenteric angiogram is typically $3 \mathrm{~mL} /$ second for a total volume of 12 to $15 \mathrm{~mL}$. It is important to seat the catheter just beyond the ostium of the mesenteric vessel; if advanced too far, early branching vessels may not be imaged on the angiogram. Glucagon $1 \mathrm{mg}$ may be administered intravenously to decrease bowel peristalsis and thereby decrease motion artifacts. Diagnostic angiography of the internal iliac arteries, which supply the middle and inferior rectal arteries, is important in cases of distal LGIB. In a series by Maleux et al, $10 \%$ of cases were caused by rectal bleeding from a branch of the internal iliac artery. ${ }^{21}$

Angiographic findings of bleeding include visualization of active contrast extravasation, pooling of contrast in the venous phase, or the presence of a pseudoaneurysm. Indirect findings can guide embolization when active bleeding is not visualized angiographically. For example, an injured vessel might be characterized by vasospasm in the absence of other direct findings. In cases of hemorrhagic neoplasm, tumoral blush and neovascularity may be identified. An early draining vein is classically seen with angiodysplasia, although the classically described "tuft" of vessels is only rarely seen. The presence of endoscopically-placed clips can also help guide angiography. Not uncommonly, trial subselection of vessels is necessary to demonstrate bleeding.

In one study, a negative bleeding focus was noted in $52 \%$ of cases, with a greater incidence in LGIB (66\%) than upper GI bleeding (46\%). ${ }^{22}$ Failure to localize a bleeding source may be attributed to slow or intermittent nature of the hemorrhage. In such cases, provocative angiography can aid in detection. Several techniques have been reported to augment bleeding temporarily and increase diagnostic sensitivity, including the endovascular administration of anticoagulants, vasodilators, and/or fibrinolytics. In a large series by Kim et al, using a combination of heparin (5000 U), nitroglycerin (200 $\mu \mathrm{g})$, and tissue plasminogen activator (titrated boluses starting at 4 $\mathrm{mg}$ ), a source of hemorrhage was uncovered in $33 \%$ of patients. ${ }^{23}$ This percentage increased to $47 \%$ when considering only those patients admitted with acute LGIB.

In cases of negative angiography, medical management should be continued. In the previously cited study, $80 \%$ of patients with a negative arteriogram had spontaneous resolution of bleeding. ${ }^{22}$ If bleeding recurs, the algorithm presented thus far should be repeated. The need for surgical intervention at any point is determined on a clinical basis.

\section{Endovascular Treatment}

The two basic endovascular treatment options are embolization and vasopressor infusion. Both aim to decrease perfusion to the site of vascular injury and thereby allow for clot formation and subsequent endogenous repair of the injured vessel. A balance must be struck with respect to the extent of embolization or vasoconstriction to prevent total devascularization of the target area and consequent bowel necrosis.

\section{Embolization}

Embolization is currently the preferred endovascular therapy in the treatment of acute LGIB. A microcatheter is deployed in a coaxial manner through a $5 \mathrm{~F}$ catheter, and the mesenteric vasculature is subselected to the level desired for embolization, preferably just proximal to the bleeding site. More distal embolization of vasculature beyond the injured vessel and closer to the capillary bed increases the risk of bowel infarction, whereas more proximal embolization (e.g., marginal artery) is less effective because blood may reach the injured vessel through mesenteric arcades. Most commonly, embolization is targeted to the level of the vasa recta or the terminal artery, although the ideal level of embolization in LGIB remains controversial. If the microcatheter cannot be advanced to at least the level of the marginal artery, we do not advise embolization because proximal colonic artery embolization can cause ischemia or infarction of a large segment of bowel. ${ }^{24}$ Compared with the upper GI tract, the lower GI tract is theorized to be at increased risk of ischemia due to the absence of significant collateral pathways. There is, however, believed to be a rich intramural vascular network that appears to offer some level of protection against bowel ischemia. 25,26

Limitations to superselective embolization include extensive atherosclerotic disease and vasospasm. These are much less of a concern with modern microcatheter technology, as evidenced by the high technical success rates of LGIB embolization in recent series. ${ }^{18,27-30}$

\section{Coils}

Microcoils are the embolic agent of choice, with a technical success rate of 81 to $100 \%{ }^{2}$ The fibers of the coil elicit thrombosis while the coil itself functions as a scaffold for thrombus. Microcoils are available in a wide selection of sizes, allowing one to correctly match the targeted vessel diameter. It is advisable to err on the side of slightly oversizing the coils 
because there is inevitably some degree of vasospasm associated with catheterization. Once the vasospasm resolves, blood flow can resume around an inadvertently undersized coil and GI hemorrhage will recur. Oversized 0.035 -inch coils may result in vessel rupture, but this is less likely to occur with similarly sized microcoils. Grossly oversized coils do not form properly within the vessel lumen and provide a less effective scaffold for thrombus. Some operators advocate nitroglycerin administration (200 $\mu \mathrm{g}$ intra-arterially) just before the pre-embolization angiogram to more accurately characterize the bleed and the caliber of the target vessel.

When the diameter of the target vessel is in doubt or there is a dubious landing zone for coil placement, detachable coils are a useful tool. The Interlock coils used in the case presentation were favored, in part, for this reason. These allow the operator to retract and reposition coils, or even completely retrieve the coil prior to final placement. They have the additional advantage of not requiring a separate pusher wire, which can be quite useful when working with limited support staff.

Compared with Gelfoam, glue, or particles, radio-opaque microcoils are easily visualized and are more precisely deployed. However, a drawback of coil embolization is that it can preclude future endovascular access to the targeted lesion. Had there been recurrent bleeding after endovascular coil placement in the previous case presentation, surgical intervention might have been the only remaining treatment option. Rebleeding rates following coil embolization have been reported between $0 \%$ and $40 \%{ }^{2}$ Fortunately, even if bleeding recurs after embolization, it is usually much less severe and the patient is more hemodynamically stable, allowing for additional surgical or endoscopic therapy. ${ }^{30}$ Repeat embolization can also be performed in cases of recurrence.

\section{Gelfoam}

As a temporary agent, Gelfoam has the theoretical advantage of allowing for future recanalization of embolized vessels. If it occurs, recanalization usually takes place within 2 to 6 weeks. However, considering bowel necrosis can occur within 8 to 12 hours in the setting of acute mesenteric ischemia, Gelfoam would not appear to provide any benefit in terms of avoiding bowel ischemia or infarction when used alone in selective embolization of LGIB. In a study of dogs, Gelfoam embolization performed at a distal level caused gross ischemic changes in the intestinal wall within 1 day. The same study found no ischemic changes when Gelfoam was applied at a proximal level (i.e., at the level of the ileal or jejunal arteries) or when coils were deployed at a distal level. ${ }^{31}$ No studies have been performed in humans to similarly compare the efficacy and risks of these embolic agents in LGIB.

Gelfoam pledgets can be administered using a microcatheter, preferably of a larger caliber ( $\geq 0.27$ inches), although smaller ones such as the Renegade STC used in the case presentation can work as well. A 1-mL syringe of saline should be kept ready to clear catheter occlusions when administering Gelfoam. Nontarget embolization is also a potential negative repercussion to using Gelfoam in this manner. Although one might conjecture that Gelfoam may be quickly and less selectively administered as pledgets from a proximal level in an emergent setting, we do not recommend this.

The "Gelfoam sandwich" technique, where Gelfoam is used as an adjunct to coil embolization, does not find common use in the lower GI tract where smaller, more distal vessels are typically targeted. Typically, placement of coils alone is sufficient to achieve intravascular stasis. Nonetheless, this technique was used in the case presentation because of the large size of the bleeding vessel as well as its location in the rectum. The dual vascular supply of rectum by the inferior mesenteric and internal iliac arteries affords a level of added protection against ischemia not seen elsewhere in the lower GI tract.

\section{Glue}

N-butyl cyanoacrylate, or "glue" (Trufill NBCA, Cordis Neurovascular, Miami Lakes, FL), works by polymerizing upon exposure to an ionic environment such as blood or saline. An immediate cast of the vessel is created causing permanent occlusion. Meticulous technique must be used because the catheter tip will occlude after one or two injections and there is a risk of catheter adherence to the vessel. Ethylene vinyl alcohol copolymer (Onyx, Micro Therapeutics) works by a similar mechanism, polymerizing upon exposure to blood, but it has the advantage of being more forgiving with respect to catheter adhesion. Catheters must be flushed with 5\% dextrose in water and dimethylsulfoxide solvent when using NBCA or Onyx, respectively, to prevent premature polymerization within the catheter. The extent of distal diffusion within the vasculature depends on the rate of injection and, in the case of NBCA, the degree of dilution with iodized oil. A greater ratio of Ethiodol to NBCA increases the polymerization time of the mixture, allowing it to travel more distally.

The primary advantage of these agents in acute LGIB is that they cause mechanical occlusion of vessels and do not rely on the patient's ability to form thrombus. Thus they are ideal agents when the patient has an uncorrected coagulopathy. Glue and Onyx also find particular utility in cases complicated by tortuous vasculature where precise delivery of coils is not possible. These liquid agents can also be delivered through smaller (0.010-inch) catheters than microcoils, which may be beneficial in small, tortuous, or spastic vessels. ${ }^{32}$ Another utility for these agents is in the embolization of pseudoaneurysms with multiple branch vessels that cannot be completely excluded with coils.

The primary concern in using glue or NBCA is that the extent of vascular penetration can be difficult to control, resulting in increased risk of ischemia and nontarget embolization. These agents require more operator experience and diligence. Recent studies for patients with LGIB have reported a $100 \%$ technical success rate with a clinical success rate of 78 to $85 \%$ using NBCA; ischemic complications occurred in 0 to $14 \%{ }^{32,33}$

\section{Particles}

Because of their small size and potential to reach the level of the intramural vasculature, particulate agents such as 
polyvinyl alcohol (PVA) or tris-acryl gelatin microspheres, are theoretically associated with an increased risk of bowel infarction. Additionally, unlike coils, particles cannot be directly visualized or precisely deposited and may also reflux into nontarget arteries. One study reported bowel infarction in $25 \%$ of patients treated with particles in conjunction with coils. $^{27}$ In a separate study, PVA particle-induced bowel ischemia resulting in hemicolectomy was also reported. ${ }^{34}$ However, several series have documented the safe use of PVA particles in treating LGIB without resultant bowel ischemia. ${ }^{35,36}$ No consensus has been obtained in the literature on the ideal particle size. In one study, smaller size particles (150 to $250 \mu \mathrm{m}$ ) were concluded to be the agent of choice, ${ }^{35}$ whereas another laboratory study found particles $<250 \mu \mathrm{m}$ to cause infarction. ${ }^{37}$ In the former study, it was postulated that particles preferentially flow along the path of least resistance toward the ruptured vessel. ${ }^{35}$ In light of the conflicting evidence, we do not advocate the use of particle embolization in most cases of LGIB.

Neoplasm-induced hemorrhage is one setting in which the use of particles is generally agreed to be safe and possibly advantageous. Although surgery is the only definitive treatment for neoplasm, particle embolization can be used as a temporizing measure in cases of emergent GI hemorrhage from primary or metastatic GI tumors. ${ }^{38}$ Particle embolization has also been successful in shrinking and devascularizing tumors prior to surgical resection. ${ }^{39}$ Use of particle sizes as small as 100 to $150 \mu \mathrm{m}$ have been reported to be technically successful and without associated bowel ischemia or postembolization syndrome. ${ }^{37}$ Care must be taken to ensure no arteriovenous shunting is present on pre-embolization selective angiography; this is especially true when using smaller particle sizes.

\section{Results}

Technical success rates of LGIB embolization now approach $100 \%,{ }^{21,27-30}$ which is attributable in large part to advances in microcatheter technology. The clinical success rate of embolization is reported as high as $90 \%$. The rate of recurrent hemorrhage after embolization is $\sim 15 \% .{ }^{1}$ However, it should be noted that rebleeding after 3 days is likely due at least in part to clinical factors such as an uncorrected coagulopathy and not to failure of the embolization technique alone. Although re-embolization for recurrent bleeding poses a theoretical increased risk of ischemic complications, it does not appear to have a negative effect on clinical outcome. ${ }^{40}$

Embolotherapy is most successful in the treatment of diverticular bleeding, with clinical success rates reported between $85 \%$ and $97 \%{ }^{41}$ In contrast, angiodysplasia appears to be relatively difficult to treat with embolization. Limited published data have shown an increased rate of early rebleeding (7 to $40 \%^{1}$ ) after embolization of angiodysplasia compared with other etiologies of LGIB. ${ }^{30,42}$ There is also some evidence that cecal bleeding is less responsive to embolization, which could be related to the increased incidence of angiodysplasia in this location.
Complications of embolotherapy include nontarget embolization resulting in bowel necrosis, ischemic stricture, arterial dissection, and contrast-induced nephrotoxicity. Even in technically successful superselective embolization, there remains a risk of ischemia. In series by Maleux et al and Kickuth et al, ischemic complications requiring surgery occurred in 5 to $10 \%$ of all cases. ${ }^{21,27}$ In a study by Bandi et al, asymptomatic mucosal ischemia occurred in $24 \%$ (although there were no clinically significant cases of intestinal ischemia). ${ }^{29}$ Most postembolization ischemic complications are clinically insignificant and do not require additional therapy. Findings of ischemia may include self-limited abdominal pain, elevated serum lactate, or asymptomatic discoloration or ulceration on endoscopy. ${ }^{37}$ Incidence of frank bowel infarction with current endovascular embolization techniques is reported to be 0 to $20 \%{ }^{2}$

\section{Vasopressin}

Vasopressin causes vasoconstriction of the smooth muscle fibers of the splanchnic blood vessels and the bowel wall. ${ }^{43}$ In this manner it decreases perfusion to the site of vascular injury to allow for clot formation. Thus, as with embolotherapy, procedural success depends on a normal coagulation cascade. Vasopressin infusion should be considered in cases where embolization is not technically achievable, such as in patients with tortuous vessels, vasospasm, or extensive atherosclerotic disease. However, it may be ineffective in the setting of extensive atherosclerosis, which impairs adequate vasoconstriction. A review of studies by Darcy reported success rates ranging from $60 \%$ to $100 \%$, with the best results obtained when treating colonic diverticular bleeding. ${ }^{33}$

In terms of angiographic technique, vasopressin infusion is much less challenging than embolotherapy. The SMA or IMA is selected with a $5 \mathrm{~F}$ angiographic catheter; a microcatheter typically is not needed. With the catheter adequately seated just beyond the vessel ostium, vasopressin is infused at a rate of $0.2 \mathrm{U} /$ minute for 20 minutes, after which angiography is repeated to assess for persistent contrast extravasation. If this rate is inadequate to stop bleeding, infusion is increased to $0.3 \mathrm{U} /$ minute or $0.4 \mathrm{U} /$ minute and another trial infusion is performed. Most operators use $0.4 \mathrm{U} /$ minute as a maximum infusion rate. Once an efficacious rate is found, it is continued for 12 hours. Subsequently, the rate is reduced by half every 12 hours and concluded with a 12-hour saline infusion. If clinical signs of active hemorrhage persist, follow-up angiography may be performed; otherwise the catheter may be removed.

Vasoconstrictor infusion is contraindicated in patients with coronary artery or cerebrovascular disease because these conditions may be exacerbated by vasopressors. Cardiovascular complications are reported to occur in 5 to $8 \%$ of patients including arrhythmias, myocardial infarction, and hypertension. ${ }^{33}$ These effects can be lessened to some degree by the concomitant systemic administration of nitroglycerin. Additional complications include catheter dislodgement with infusion into a nontarget artery and thrombosis secondary to prolonged catheterization. Overaggressive treatment with vasopressin can also lead to mesenteric artery thrombosis and bowel infarction. It has been suggested that infusion of 
vasopressin after previous embolization could cause ischemia by affecting the collateral supply to the bowel. Because of these potential complications, at our institution intensive care unit admission is required when using vasopressor therapy.

Rebleeding is a commonly cited drawback to vasopressin use, with rates ranging between $36 \%$ and $43 \%{ }^{33}$ It is suspected to occur when the vasospasm-induced thrombus resolves before the underlying vascular lesion heals. The recurrence rate is higher with vasopressin infusion compared with embolotherapy.

With improved microcatheter technology, varied embolization options, as well as preference for immediate angiographic result, the use of vasopressin has waned, and there is a paucity of recent literature regarding this technique. Nevertheless, this technique may be used in place of embolization for diffuse lesions and when superselective embolization cannot be achieved. ${ }^{33}$

\section{Surgery}

Surgery is more often required for lower than upper GI bleeding despite the relatively increased incidence of upper GI hemorrhage. ${ }^{44}$ About $5 \%$ of patients require emergent surgery for acute massive LGIB. ${ }^{45}$ Because of the associated morbidity and mortality, surgery is reserved as the last resort in controlling LGIB. ${ }^{46}$ Mortality of emergent segmental colonic resection is reported to be $5 \%$ in localized $\mathrm{LGIB}^{47}$ and $33 \%$ in blind nonlocalized colon resection. ${ }^{46}$ Overall mortality after surgery for LGIB is reported as 15 to $30 \%$, higher than is reported for surgical mortality for upper GI bleeding.

Even greater morbidity and mortality rates have been noted in after-hours emergent surgery in elderly patients. ${ }^{48,49}$ There may be a role for embolization used specifically to stabilize patients prior to emergent bowel resection. To this end, we are occasionally called on at our institution to embolize an entire segment of bowel in patients with LGIB. In this situation, administration of Gelfoam slurry from a relatively proximal position, or central coil embolization, may be considered as a temporizing measure in patients who will undergo surgery the next morning.

If the source of bleeding remains uncertain and/or no endovascular treatments can be safely employed, injection of methylene blue intraoperatively via a catheter placed fluoroscopically may help the surgeon in localizing the bleeding source.

\section{Teaching Points/Pearls}

1. Knowledge of the pre- and postangiographic evaluation in addition to the endovascular therapies available is paramount in optimizing care of the patient with LGIB.

2. Although coils are the mainstay of endovascular therapy for LGIB, Gelfoam, glue, and particles have unique roles.

3. Embolization does not treat the underlying pathology related to LGIB; postendovascular colonoscopy is necessary in nearly all cases.

\section{References}

1 Barnert J, Messmann H. Diagnosis and management of lower gastrointestinal bleeding. Nat Rev Gastroenterol Hepatol 2009; 6(11):637-646

2 d'Othée BJ, Surapaneni P, Rabkin D, Nasser I, Clouse M. Microcoil embolization for acute lower gastrointestinal bleeding. Cardiovasc Intervent Radiol 2006;29(1):49-58

3 Koh DC, Luchtefeld MA, Kim DG, et al. Efficacy of transarterial embolization as definitive treatment in lower gastrointestinal bleeding. Colorectal Dis 2009;11(1):53-59

4 Zuckerman GR, Prakash C. Acute lower intestinal bleeding: part I: clinical presentation and diagnosis. Gastrointest Endosc 1998;48 (6):606-617

5 Longstreth GF. Epidemiology and outcome of patients hospitalized with acute lower gastrointestinal hemorrhage: a populationbased study. Am J Gastroenterol 1997;92(3):419-424

6 Strate LL. Lower GI bleeding: epidemiology and diagnosis. Gastroenterol Clin North Am 2005;34(4):643-664

7 Stark ME, Gostout CJ, Balm RK. Clinical features and endoscopic management of Dieulafoy's disease. Gastrointest Endosc 1992; 38(5):545-550

8 Lee YT, Walmsley RS, Leong RW, Sung JJ. Dieulafoy's lesion. Gastrointest Endosc 2003;58(2):236-243

9 Laine L, Shah A. Randomized trial of urgent vs. elective colonoscopy in patients hospitalized with lower GI bleeding. Am J Gastroenterol 2010;105(12):2636-2641; quiz 2642

10 Billingham RP. The conundrum of lower gastrointestinal bleeding. Surg Clin North Am 1997;77(1):241-252

11 Green BT, Rockey DC, Portwood G, et al. Urgent colonoscopy for evaluation and management of acute lower gastrointestinal hemorrhage: a randomized trial. Am J Gastroenterol 2005;100(11): 2395-2402

12 Valera JM, Pino RQ, Poniachik J, et al. Endoscopic band ligation of bleeding Dieulafoy lesions: the best therapeutic strategy. Endoscopy 2006;38(2):193-194

13 Ahn DW, Lee SH, Park YS, et al. Hemostatic efficacy and clinical outcome of endoscopic treatment of Dieulafoy's lesions: comparison of endoscopic hemoclip placement and endoscopic band ligation. Gastrointest Endosc 2012;75(1):32-38

14 Gunderman R, Leef J, Ong K, Reba R, Metz C. Scintigraphic screening prior to visceral arteriography in acute lower gastrointestinal bleeding. J Nucl Med 1998;39(6):1081-1083

15 Millward SF. ACR appropriateness criteria on treatment of acute nonvariceal gastrointestinal tract bleeding. J Am Coll Radiol 2008;5(4):550-554

16 Ng DA, Opelka FG, Beck DE, et al. Predictive value of technetium Tc 99m-labeled red blood cell scintigraphy for positive angiogram in massive lower gastrointestinal hemorrhage. Dis Colon Rectum 1997;40(4):471-477

17 Martí M, Artigas JM, Garzón G, Alvarez-Sala R, Soto JA. Acute lower intestinal bleeding: feasibility and diagnostic performance of CT angiography. Radiology 2012;262(1):109-116

18 American College of Radiology (ACR) Committee on Drugs and Contrast Media. ACR Manual on Contrast Media: Version 7, 2010. Available at: xray.ufl.edu/files/2008/06/FullManualACRContrastVersion7.pdf

19 Schenker MP, Duszak R Jr, Soulen MC, et al. Upper gastrointestinal hemorrhage and transcatheter embolotherapy: clinical and technical factors impacting success and survival. J Vasc Interv Radiol 2001;12(11):1263-1271

20 Aina R, Oliva VL, Therasse E, et al. Arterial embolotherapy for upper gastrointestinal hemorrhage: outcome assessment. J Vasc Interv Radiol 2001;12(2):195-200

21 Maleux G, Roeflaer F, Heye S, et al. Long-term outcome of transcatheter embolotherapy for acute lower gastrointestinal hemorrhage. Am J Gastroenterol 2009;104(8):2042-2046 
$22 \mathrm{Kim} \mathrm{JH}$, Shin JH, Yoon HK, et al. Angiographically negative acute arterial upper and lower gastrointestinal bleeding: incidence, predictive factors, and clinical outcomes. Korean J Radiol 2009;10(4):384-390

23 Kim CY, Suhocki PV, Miller MJ Jr, Khan M, Janus G, Smith TP. Provocative mesenteric angiography for lower gastrointestinal hemorrhage: results from a single-institution study. J Vasc Interv Radiol 2010;21(4):477-483

24 Krämer SC, Görich J, Rilinger N, et al. Embolization for gastrointestinal hemorrhages. Eur Radiol 2000;10(5):802-805

25 Suh SW, Choi YS, Kim H, Kim BG. Small bowel ischemia after angiographic embolization for angiodysplasia of lower gastrointestinal tract: the case for conservative management. Clin Res Hepatol Gastroenterol 2011;35(12):819-822

26 Kuo WT, Lee DE, Saad WE, Patel N, Sahler LG, Waldman DL. Superselective microcoil embolization for the treatment of lower gastrointestinal hemorrhage. J Vasc Interv Radiol 2003;14(12): 1503-1509

27 Kickuth R, Rattunde H, Gschossmann J, Inderbitzin D, Ludwig K, Triller J. Acute lower gastrointestinal hemorrhage: minimally invasive management with microcatheter embolization. J Vasc Interv Radiol 2008;19(9):1289-1296, e2

28 Kwak HS, Han YM, Lee ST. The clinical outcomes of transcatheter microcoil embolization in patients with active lower gastrointestinal bleeding in the small bowel. Korean J Radiol 2009;10(4): 391-397

29 Bandi R, Shetty PC, Sharma RP, Burke TH, Burke MW, Kastan D. Superselective arterial embolization for the treatment of lower gastrointestinal hemorrhage. J Vasc Interv Radiol 2001;12(12): 1399-1405

30 Funaki B, Kostelic JK, Lorenz J, et al. Superselective microcoil embolization of colonic hemorrhage. AJR Am J Roentgenol 2001;177(4):829-836

31 Han YM, Lee JM, Jin KY, Lee SY, Kim CS. Embolization of superior mesenteric artery branches in dogs. Ischemic bowel changes depend on location of vessel occlusion and embolic materials. Invest Radiol 1999;34(10):629-635

32 Frodsham A, Berkmen T, Ananian C, Fung A. Initial experience using N-butyl cyanoacrylate for embolization of lower gastrointestinal hemorrhage. J Vasc Interv Radiol 2009;20(10):1312-1319

33 Darcy M. Treatment of lower gastrointestinal bleeding: vasopressin infusion versus embolization. J Vasc Interv Radiol 2003; 14(5):535-543

34 DeBarros J, Rosas L, Cohen J, Vignati P, Sardella W, Hallisey M. The changing paradigm for the treatment of colonic hemorrhage: superselective angiographic embolization. Dis Colon Rectum 2002;45(6):802-808

35 Defreyne L, Vanlangenhove P, De Vos M, et al. Embolization as a first approach with endoscopically unmanageable acute nonvariceal gastrointestinal hemorrhage. Radiology 2001;218(3):739-748
36 Guy GE, Shetty PC, Sharma RP, Burke MW, Burke TH. Acute lower gastrointestinal hemorrhage: treatment by superselective embolization with polyvinyl alcohol particles. AJR Am J Roentgenol 1992;159(3):521-526

37 Kusano S, Murata K, Ohuchi H, Motohashi O, Atari H. Low-dose particulate polyvinyl alcohol embolization in massive small artery intestinal hemorrhage. Experimental and clinical results. Invest Radiol 1987;22(5):388-392

38 Fidelman N, Freed RC, Nakakura EK, Rosenberg J, Bloom AI. Arterial embolization for the management of gastrointestinal hemorrhage from metastatic renal cell carcinoma. J Vasc Interv Radiol 2010; 21(5):741-744

39 Kurihara N, Kikuchi K, Tanabe M, et al. Partial resection of the second portion of the duodenum for gastrointestinal stromal tumor after effective transarterial embolization. Int J Clin Oncol 2005;10(6):433-437

40 Mensel B, Kühn JP, Kraft M, et al. Selective microcoil embolization of arterial gastrointestinal bleeding in the acute situation: outcome, complications, and factors affecting treatment success. Eur J Gastroenterol Hepatol 2012;24(2):155-163

41 Khanna A, Ognibene SJ, Koniaris LG. Embolization as first-line therapy for diverticulosis-related massive lower gastrointestinal bleeding: evidence from a meta-analysis. J Gastrointest Surg 2005;9(3):343-352

42 Peck DJ, McLoughlin RF, Hughson MN, Rankin RN. Percutaneous embolotherapy of lower gastrointestinal hemorrhage. J Vasc Interv Radiol 1998;9(5):747-751

43 Cynamon J, Atar E, Steiner A, et al. Catheter-induced vasospasm in the treatment of acute lower gastrointestinal bleeding. J Vasc Interv Radiol 2003;14(2 Pt 1):211-216

44 Peura DA, Lanza FL, Gostout CJ, Foutch PG. The American College of Gastroenterology Bleeding Registry: preliminary findings. Am J Gastroenterol 1997;92(6):924-928

45 Al Qahtani AR, Satin R, Stern J, Gordon PH. Investigative modalities for massive lower gastrointestinal bleeding. World J Surg 2002; 26(5):620-625

46 Setya V, Singer JA, Minken SL. Subtotal colectomy as a last resort for unrelenting, unlocalized, lower gastrointestinal hemorrhage: experience with 12 cases. Am Surg 1992;58(5):295-299

47 Pennoyer WP, Vignati PV, Cohen JL. Management of angiogram positive lower gastrointestinal hemorrhage: long term follow-up of non-operative treatments. Int J Colorectal Dis 1996;11(6): 279-282

48 Griner D, Adams A, Kotwall CA, Clancy TV, Hope WW. After-hours urgent and emergent surgery in the elderly: outcomes and prognostic factors. Am Surg 2011;77(8):1021-1024

49 Kurian A, Suryadevara S, Ramaraju D, et al. In-hospital and 6month mortality rates after open elective vs open emergent colectomy in patients older than 80 years. Dis Colon Rectum 2011;54(4):467-471 